\title{
Supporting Information for Clusters in Liquid Fatty Acids: Structure and Role in Nucleation
}

\author{
John A. Noël, ${ }^{a}$ Luc M. LeBlanc, ${ }^{a}$ Daphne Sunita Patterson, ${ }^{b}$ Laurent Kreplak, ${ }^{\mathrm{c}, \mathrm{d}}$ \\ Michael Fleischauer, ${ }^{b, e}$ Erin R. Johnson ${ }^{a}$ and Mary Anne White, a,c,d,
}

${ }^{a}$ Department of Chemistry, Dalhousie University, Halifax, Nova Scotia, B3H 4R2, Canada

${ }^{b}$ National Research Council - Nanotechnology Research Centre, Edmonton, Alberta, T6G 2M9, Canada

${ }^{c}$ Department of Physics and Atmospheric Science, Dalhousie University, Halifax, Nova Scotia, B3H 4R2, Canada

${ }^{d}$ Clean Technology Research Institute, Dalhousie University, Halifax, Nova Scotia, B3H 4R2, Canada

${ }^{\text {e }}$ Department of Physics, University of Alberta, Edmonton, Alberta, T6G 2E1, Canada

*Corresponding author; email: mawhite@dal.ca 


\section{S.1. Supercooling in Analogous Materials}

The responses to thermal cycling of three additional, non-fatty acid, 12-carbon molecular materials (1-dodecanol $\left[\mathrm{H}_{3} \mathrm{C}\left(\mathrm{CH}_{2}\right)_{10} \mathrm{CH}_{2} \mathrm{OH}\right]$, dodecanamide $\left[\mathrm{H}_{3} \mathrm{C}\left(\mathrm{CH}_{2}\right){ }_{10} \mathrm{C}(\mathrm{O}) \mathrm{NH}_{2}\right]$, and dodecanedioic acid $\left.\left[\mathrm{HOC}(\mathrm{O})\left(\mathrm{CH}_{2}\right)_{10} \mathrm{C}(\mathrm{O}) \mathrm{OH}\right]\right)$ were compared to the response of dodecanoic acid, as reported earlier. ${ }^{1}$ This set of experiments was carried out to test the generality of the induced supercooling observed in fatty acids to other similar materials, and to provide further insights on the role of clusters in the liquid state.

\section{S.1.1. Materials and Method}

Thermal cycling of PCMs (1-dodecanol [Aldrich, 98 \%]; dodecanamide [Toyko Chemical Industry, > $96 \%$ ]; dodecanedioic acid [Alfa Aesar, $99 \%$ ]) was performed using differential scanning calorimetry (DSC; TA Instruments DSC Q200), calibrated with the melting of indium before each set of measurements. DSC experiments were made using a $2 \mathrm{~K} \mathrm{\text {min}^{-1 }}$ scanning rate under a $25 \mathrm{~mL} \mathrm{~min}^{-1}$ flow of helium. Sample masses were 3 to $8 \mathrm{mg}$, and samples were contained in crimped aluminum DSC pans. Melting and crystallization temperatures were taken as the onset temperature. Each sample was heated past the melting point $\left(T_{\text {fus }}\right)$ to a temperature higher than $T_{\text {fus }}$, and held isothermally at that temperature for $60 \mathrm{~min}$. The sample was then cooled until crystallization occurred. The process was then repeated with increasing holding temperatures, following the scheme described elsewhere. ${ }^{1}$

\section{S.1.2. Dodecanamide}

Dodecanamide $\left(T_{f u s}=98 \pm 1^{\circ} \mathrm{C}\right)$ behaved similarly to dodecanoic acid under thermal cycling (Figure S1): it required little supercooling to initiate crystallization when heated to temperatures only moderately above $T_{f u s}$. When heated to temperatures more than $50 \mathrm{~K}$ above its $T_{f u s}$, dodecanamide began to exhibit increased supercooling. However, the magnitude of 
supercooling continued to rise in the range of accessible temperatures, and did not plateau as it did for dodecanoic acid. (Dodecanamide decomposed when it was heated to a temperature more than $85 \mathrm{~K}$ above its $T_{\text {fus }}$.)

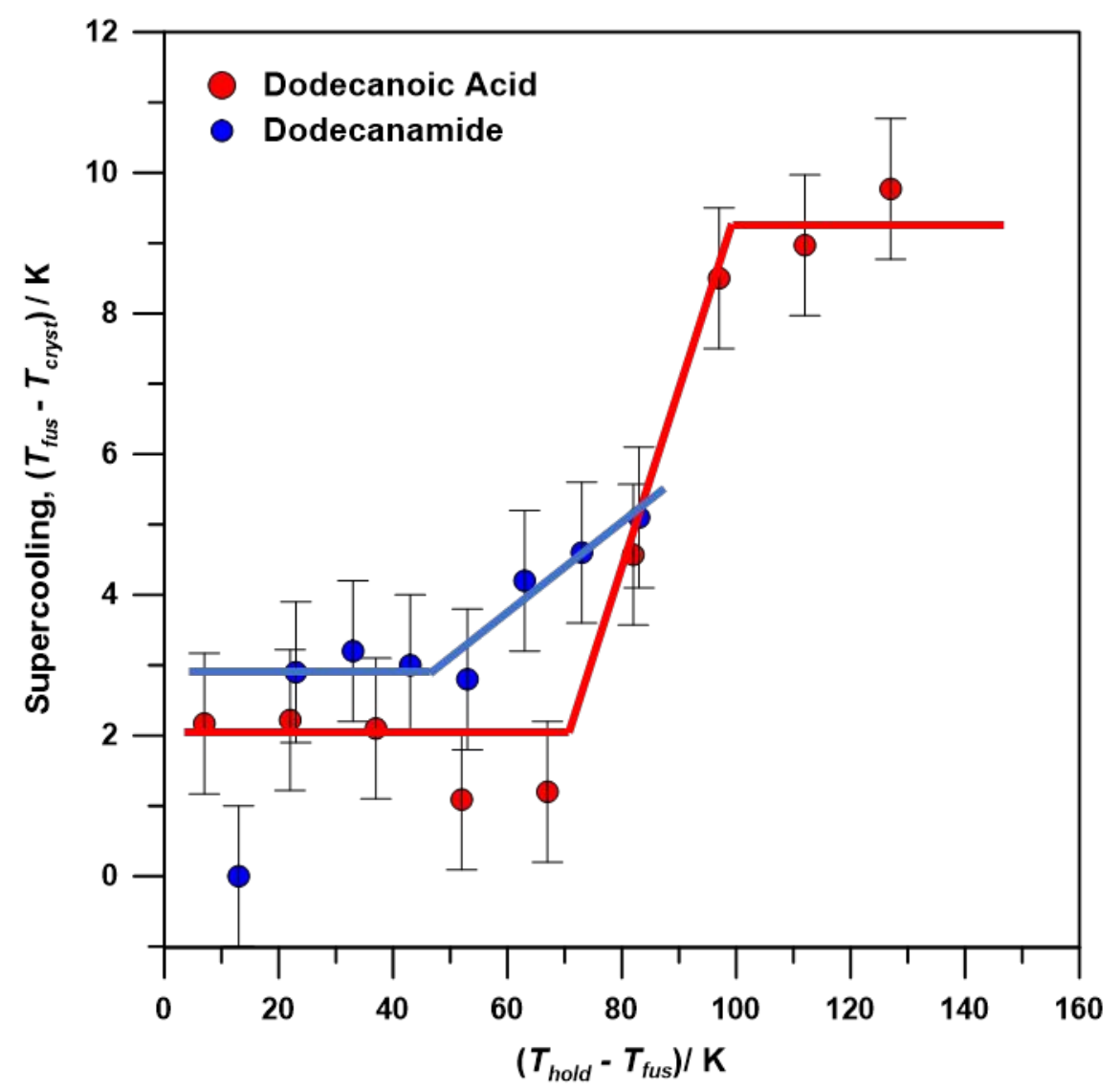

Figure S1. Comparison of the results of the thermal cycling experiments for dodecanoic acid $\left(T_{\text {fus }}=43{ }^{\circ} \mathrm{C}\right)$ and dodecanamide $\left(T_{\text {fus }}=98^{\circ} \mathrm{C}\right)$.

Dodecanoic acid and dodecanamide have similar head-group structures; both have a carbonyl group and an additional hydrogen-bond donor/acceptor group (-OH for dodecanoic acid and $-\mathrm{NH}_{2}$ for dodecanamide). Their similar molecular structures and behavior under thermal cycling suggest that they also might have similar liquid-phase structures. As such, nucleation of dodecanamide could proceed via a similar mechanism as the nucleation of dodecanoic acid. 
As an aside, we note that $\Delta_{f u s} H$ of dodecanamide is $180 \mathrm{~J} \mathrm{~g}^{-1}$, essentially the same value as for dodecanoic acid, ${ }^{2}$ and therefore it could be a useful PCM, provided the temperature is kept below its decomposition point of $180^{\circ} \mathrm{C}$.

\section{S.1.3. 1-Dodecanol}

Unlike dodecanoic acid, 1-dodecanol $\left(T_{f u s}=21 \pm 1{ }^{\circ} \mathrm{C}\right)$ did not supercool (Figure S2), even when heated as high as $125 \mathrm{~K}$ above its $T_{\text {fus }}$. Fatty alcohols have been reported to form oligomeric structures in the liquid phase ${ }^{3,4}$ suggested to be cyclic tetramers. ${ }^{5}$

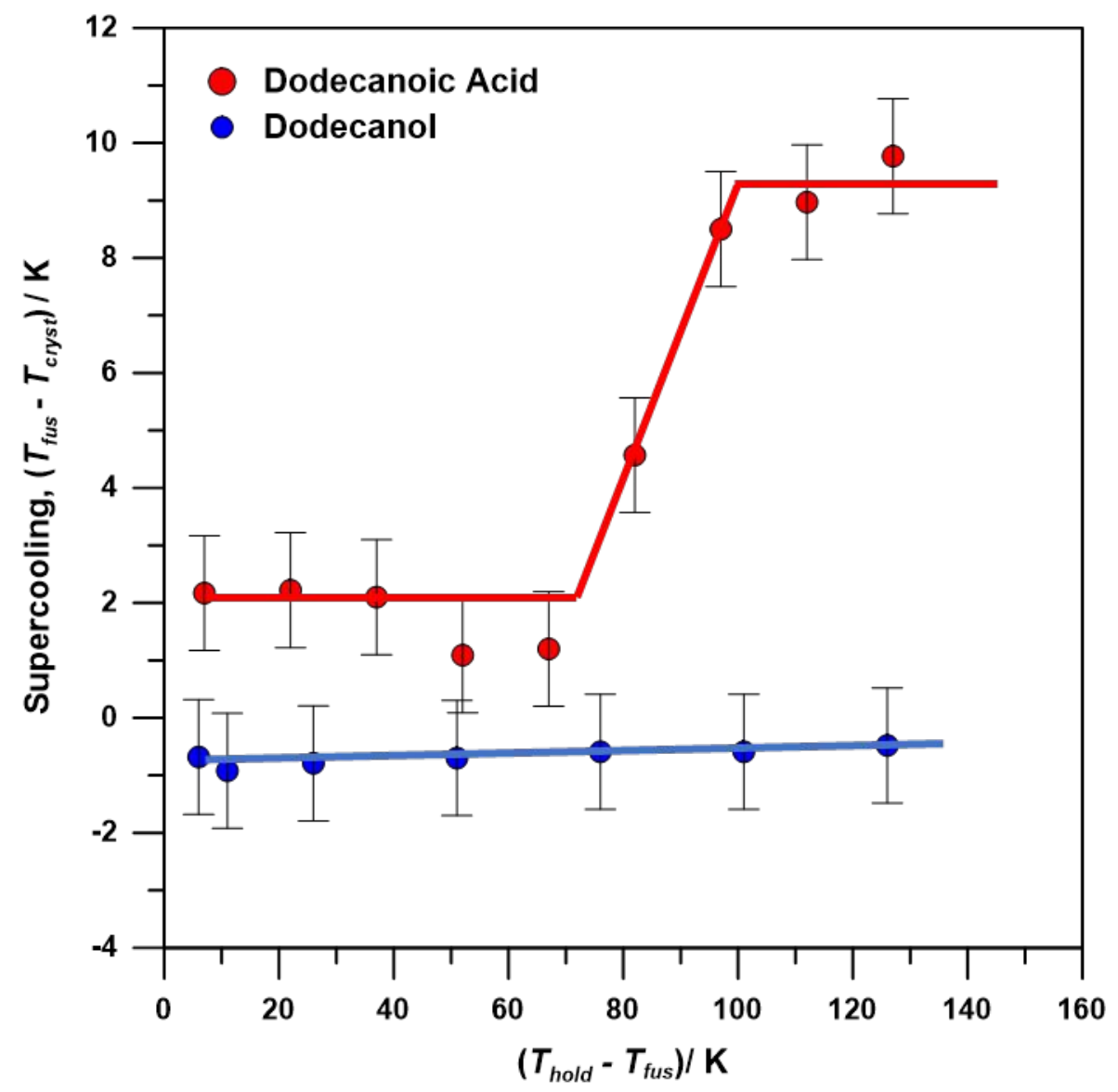

Figure S2. Comparison of the results of the thermal cycling experiments for dodecanoic acidError! Bookmark not defined. $\left(T_{f u s}=43^{\circ} \mathrm{C}\right)$ and 1 -dodecanol $\left(T_{f u s}=21^{\circ} \mathrm{C}\right)$. 
The SAXS pattern of 1-dodecanol produced a diffraction peak centered at $q \approx 0.32 \AA^{-1}(d$ $=19.6 \AA)$. The peak was symmetrical and was well-fit with a single Gaussian function. This peak was attributed to the short-range structure of the bulk liquid; no clusters were detected. The $d$-spacing of the peak is similar to the longitudinal spacing found for the bulk liquid in dodecanoic acid. As such, 1-dodecanol and dodecanoic acid might have similar short-range ordering in their liquid phases. The peak position for dodecanol stayed relatively constant on heating, but the peak area decreased substantially (Figure S3). The diffraction peak attributed to the bulk liquid in dodecanoic acid did not decrease in area with increasing temperature; the liquid maintained its short-range ordering. The decrease in the peak area for dodecanol suggests that the proportion of short-range ordered molecules decreased with increasing temperature. This could be due to a change in the hydrogen bonding of liquid dodecanol. Dodecanoic acid has two strong hydrogen bonds through the carboxyl groups holding together the dimeric units. With only a single hydroxyl group, it could be easier to separate the weaker hydrogen bonds in dodecanol. ${ }^{6}$ Long-chain alcohol monomers would likely orient themselves differently than dimers or any oligomeric structure, and so the area of the peak associated with the short-range ordering of the molecules at low temperature decreased, likely due to an increased proportion of monomeric dodecanol molecules. 


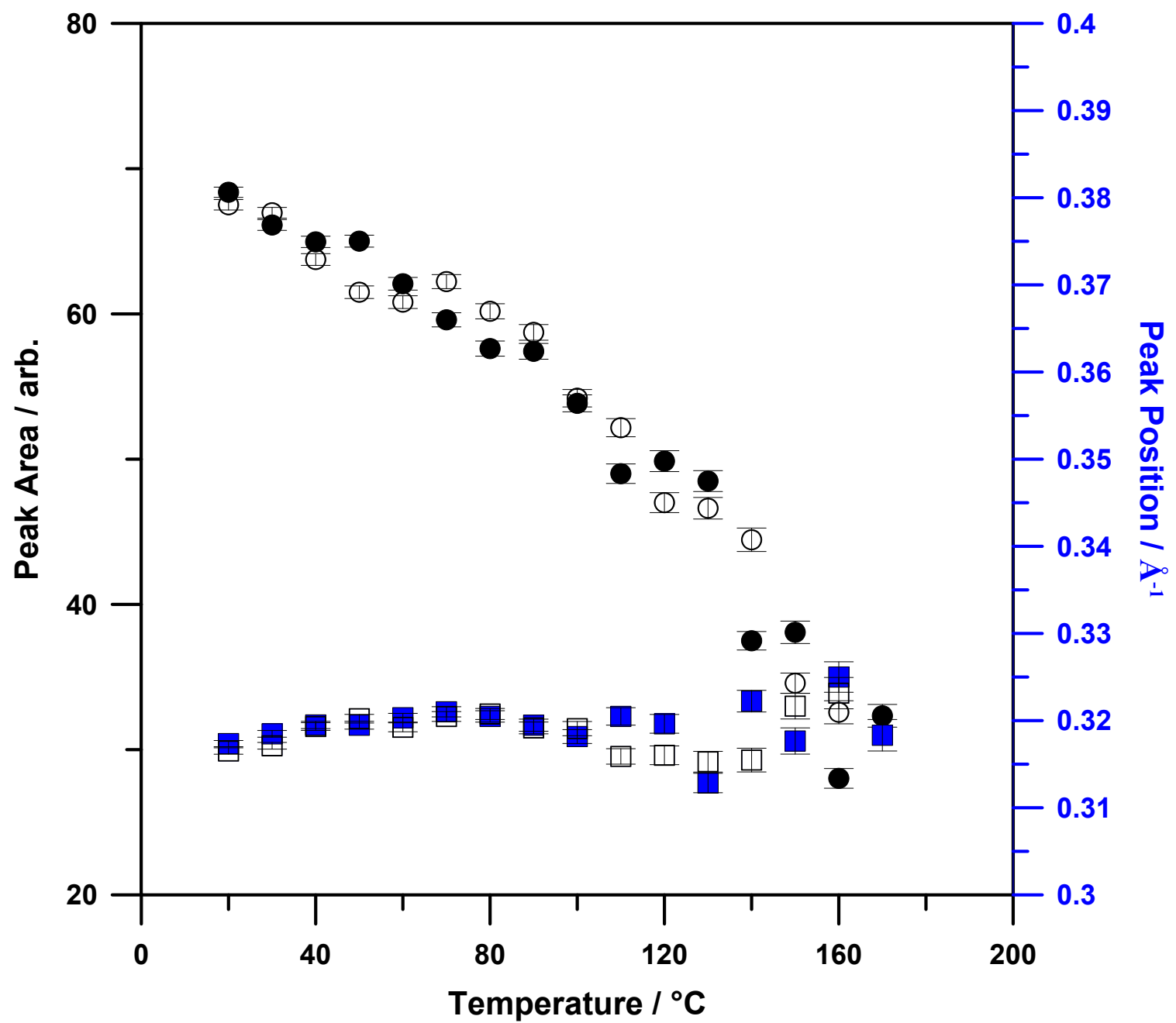

Figure S3. Area (circles) and position (squares) of the diffraction peak in the SAXS pattern of 1dodecanol $\left(T_{f u s}=21^{\circ} \mathrm{C}\right)$ on heating (closed) and on cooling (open).

On cooling, no hysteresis was observed in the dodecanol diffraction peak area.

Monomeric dodecanol molecules should be more mobile in the liquid phase than dodecanoic acid dimers. Disruptions to the ordering in liquid dodecanol could be easily repaired, explaining why thermal treatment did not induce supercooling in dodecanol.

Note that dodecanol has a high $\Delta_{f u s} H$ of $206 \mathrm{~J} \mathrm{~g}^{-1}$, especially large for its low melting temperature of $21^{\circ} \mathrm{C}$. These favourable factors, combined with its resistance to supercooling, make dodecanol a promising PCM. 


\section{S.1.4. Dodecanedioic Acid}

Dodecanedioic acid $\left(T_{f u s}=128 \pm 1{ }^{\circ} \mathrm{C}\right)$ has carboxyl groups on both ends of the molecule, each capable of forming strong hydrogen bonds. The supercooling needed for nucleation of dodecanedioic acid increased linearly with the holding temperature from the lowest temperatures examined (Figure S4), with no temperature range with only minor supercooling as there had been for dodecanoic acid. While the mobility of 1-dodecanol might have prevented its supercooling, lack of mobility in dodecanedioic acid could be the cause of its supercooling. Presuming that dodecanedioic acid forms oligomeric chains, which is reasonable given its hydrogen bonding on both ends, the chains could become tangled or intertwined. This would inhibit reorientation of the molecules into the appropriate configuration for crystallization, leading to supercooling. 


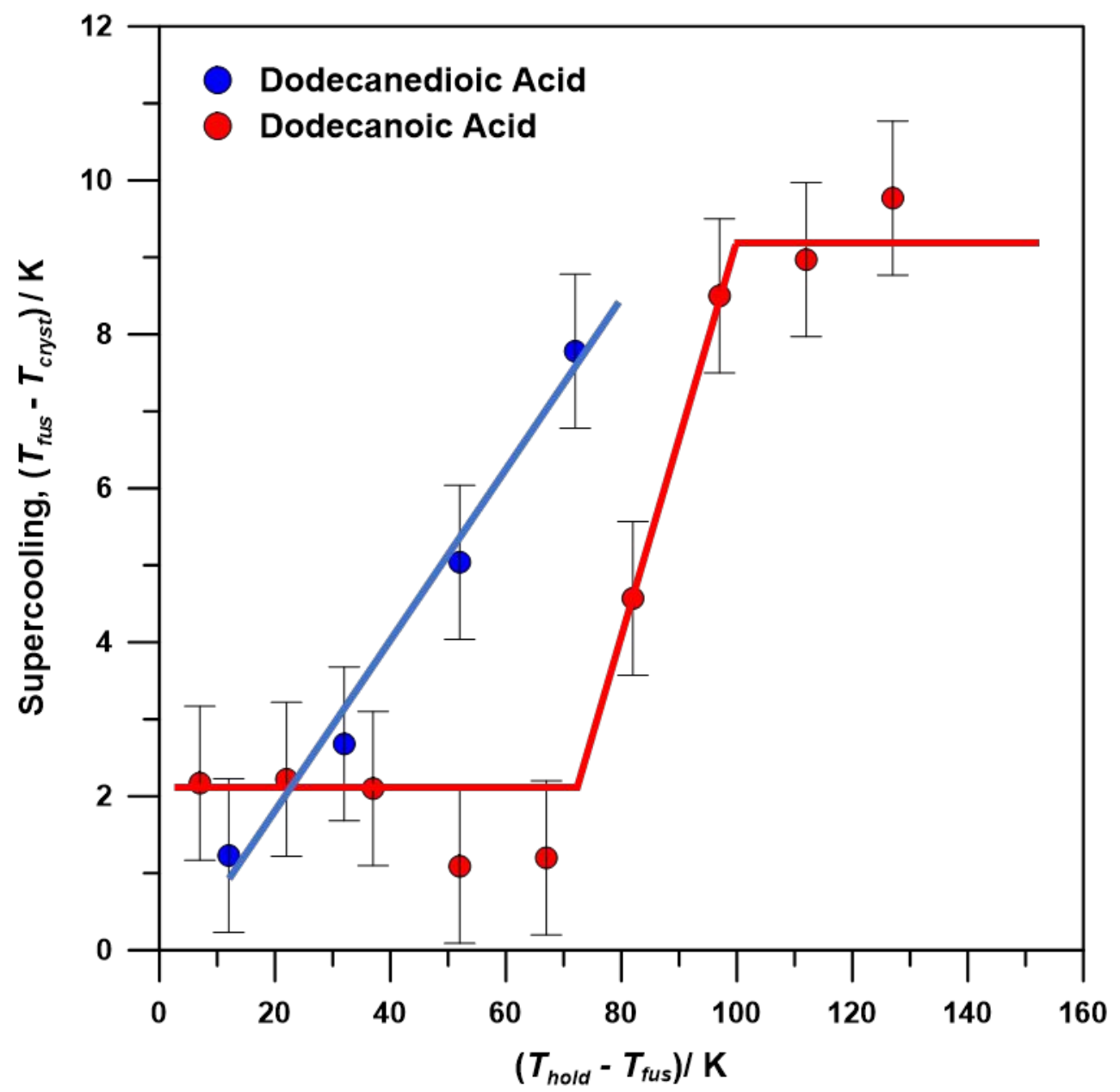

Figure S4. Comparison of the results of the thermal cycling experiments on dodecanoic acidError! Bookmark not defined. $\left(T_{\text {fus }}=43{ }^{\circ} \mathrm{C}\right)$ and on dodecanedioic acid $\left(T_{\text {fus }}=128^{\circ} \mathrm{C}\right)$.

While dodecanoic acid has a high value of $\Delta_{f u s} H\left(216 \mathrm{~J} \mathrm{~g}^{-1}\right)$, its propensity to supercool could present challenges with regard to implementation as a PCM in thermal energy storage applications. However, if the maximum temperature of its application were limited to $138{ }^{\circ} \mathrm{C}$, it could be a very useful moderate-temperature PCM. 


\section{S.2. Additional SAXS Results}

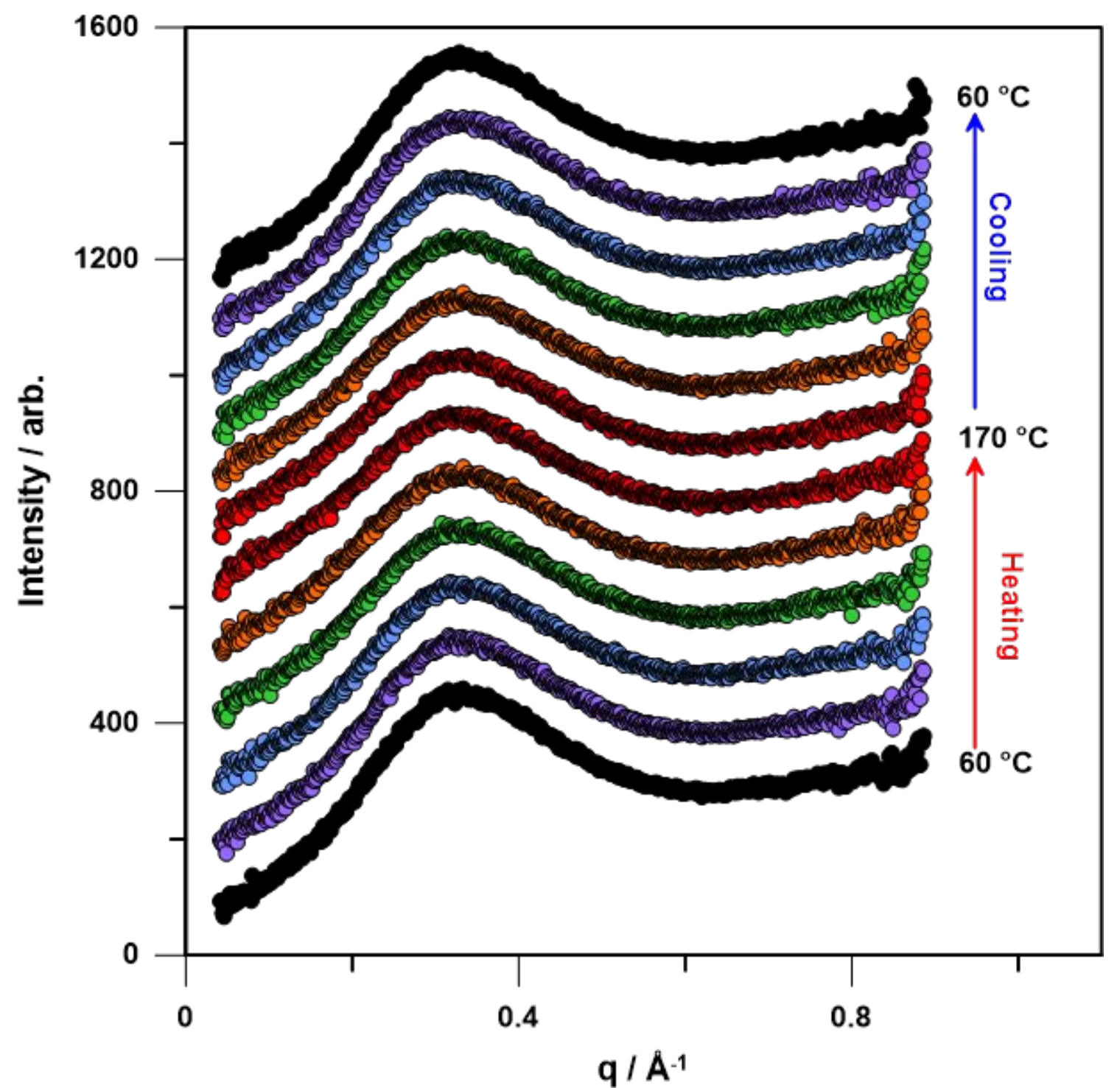

Figure S5. SAXS patterns for dodecanoic acid on heating and on cooling at $20 \mathrm{~K}$ increments. Curves are offset for clarity and data have been thinned to show only half of the temperatures at which measurements were taken, and only every $20^{\text {th }}$ point. 


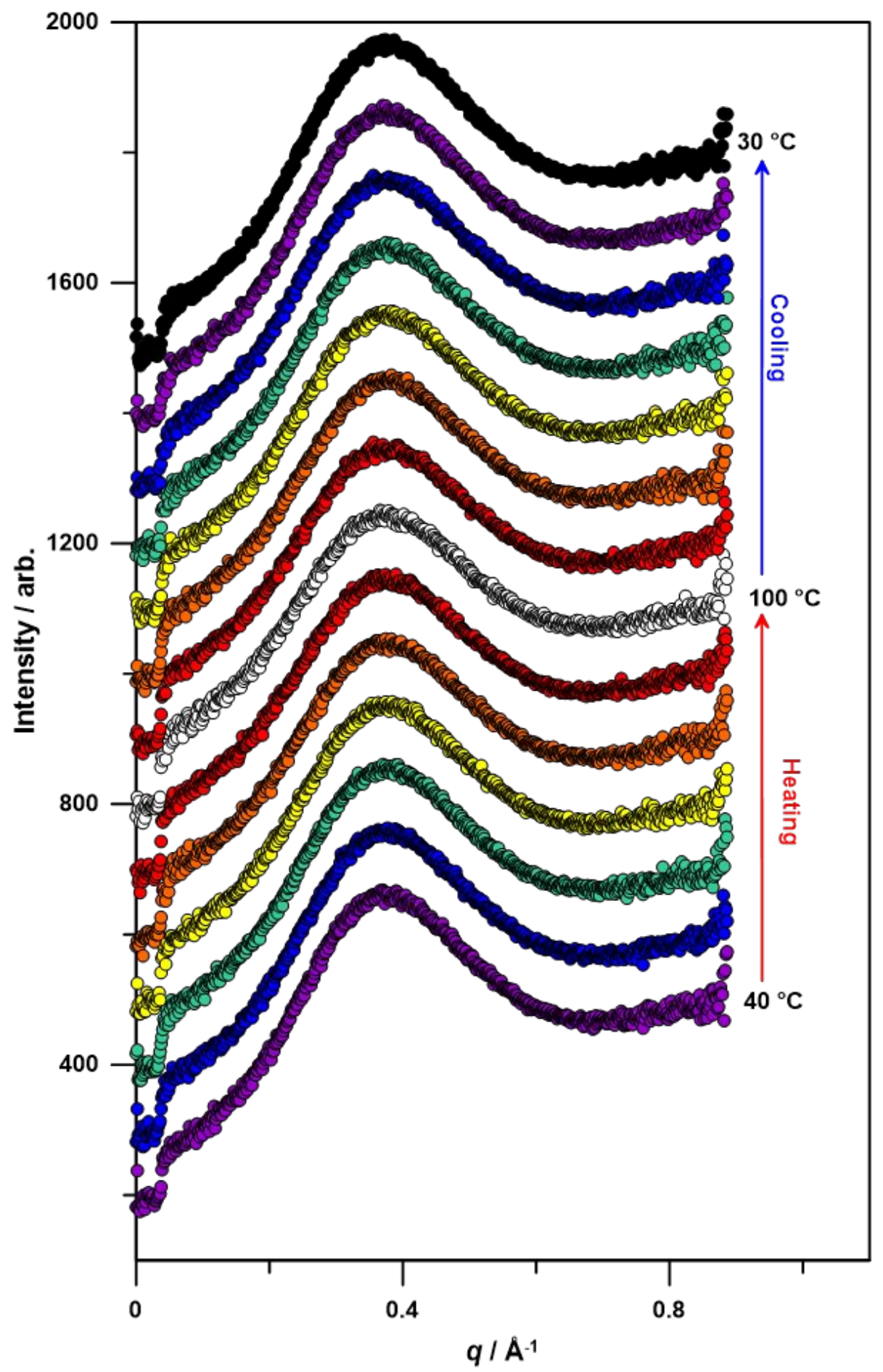

Figure S6. SAXS patterns for decanoic acid on heating and on cooling at $10 \mathrm{~K}$ increments. Curves are offset for clarity and data have been thinned to show every $20^{\text {th }}$ point. 


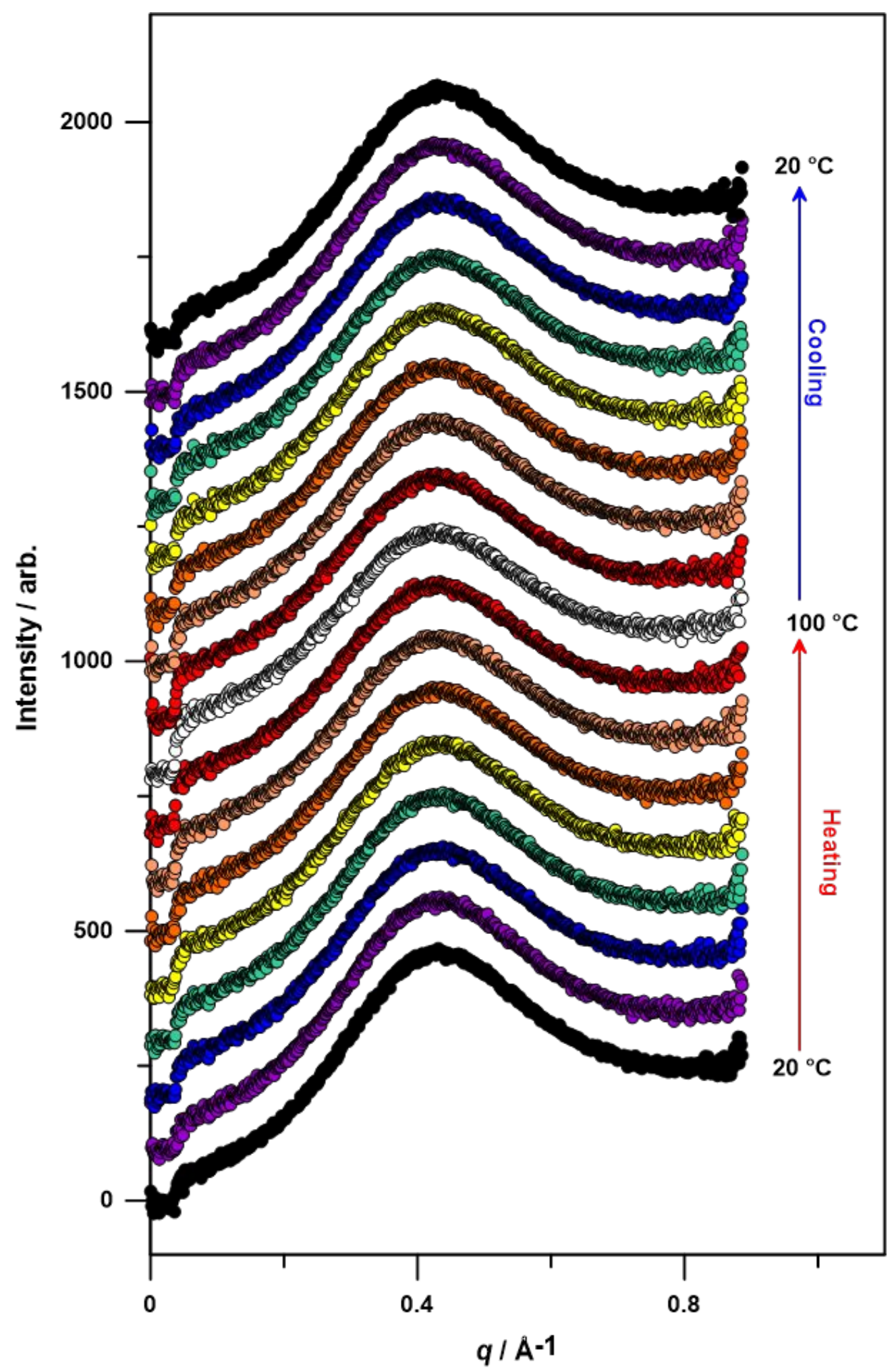

Figure S7. SAXS patterns for octanoic acid on heating and on cooling at $10 \mathrm{~K}$ increments. Curves are offset for clarity and data have been thinned to show every $20^{\text {th }}$ point. 


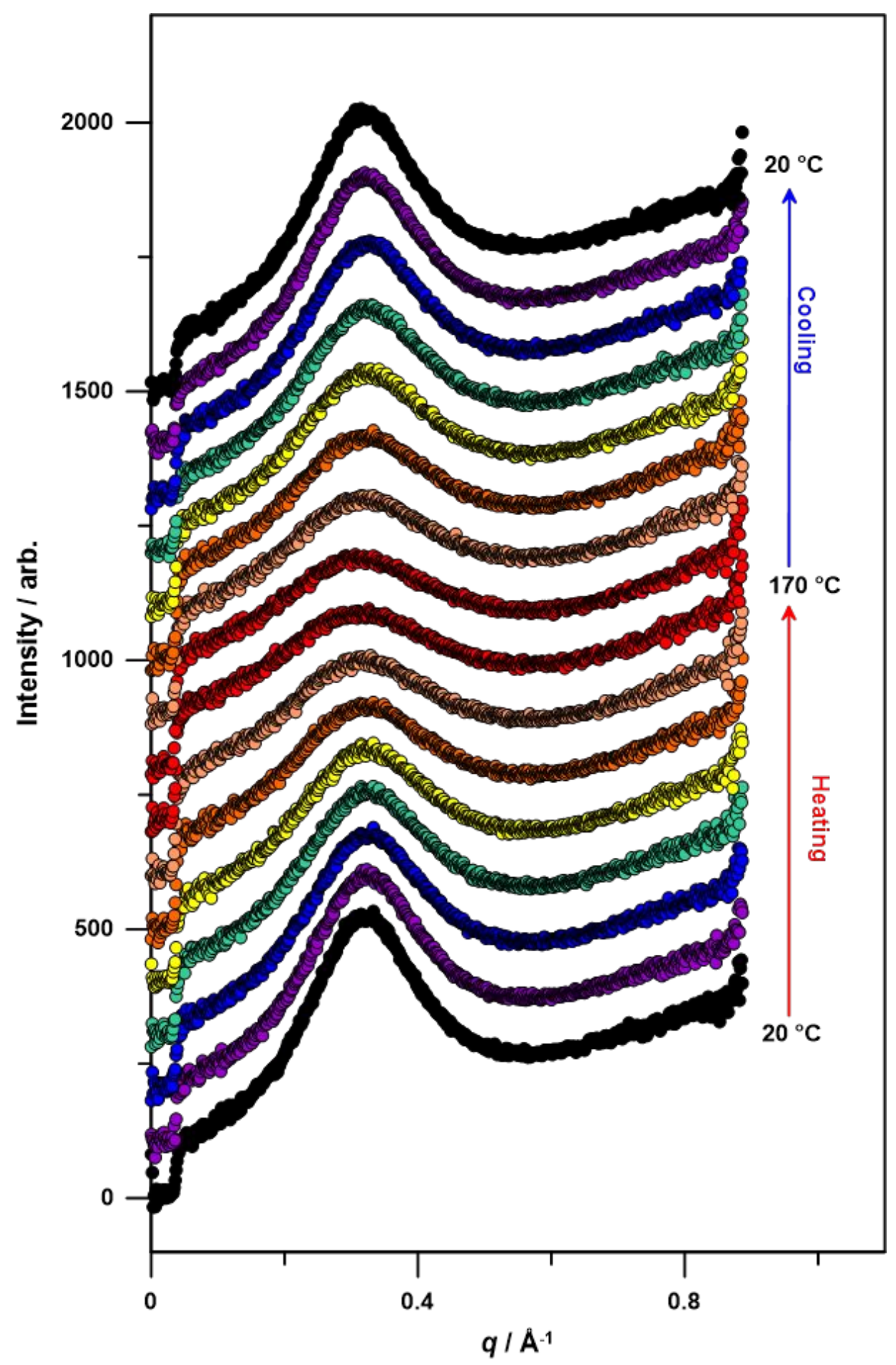

Figure S8. SAXS patterns for 1-dodecanol on heating and on cooling at $20 \mathrm{~K}$ increments. Curves are offset for clarity and data have been thinned to show every $20^{\text {th }}$ point. 


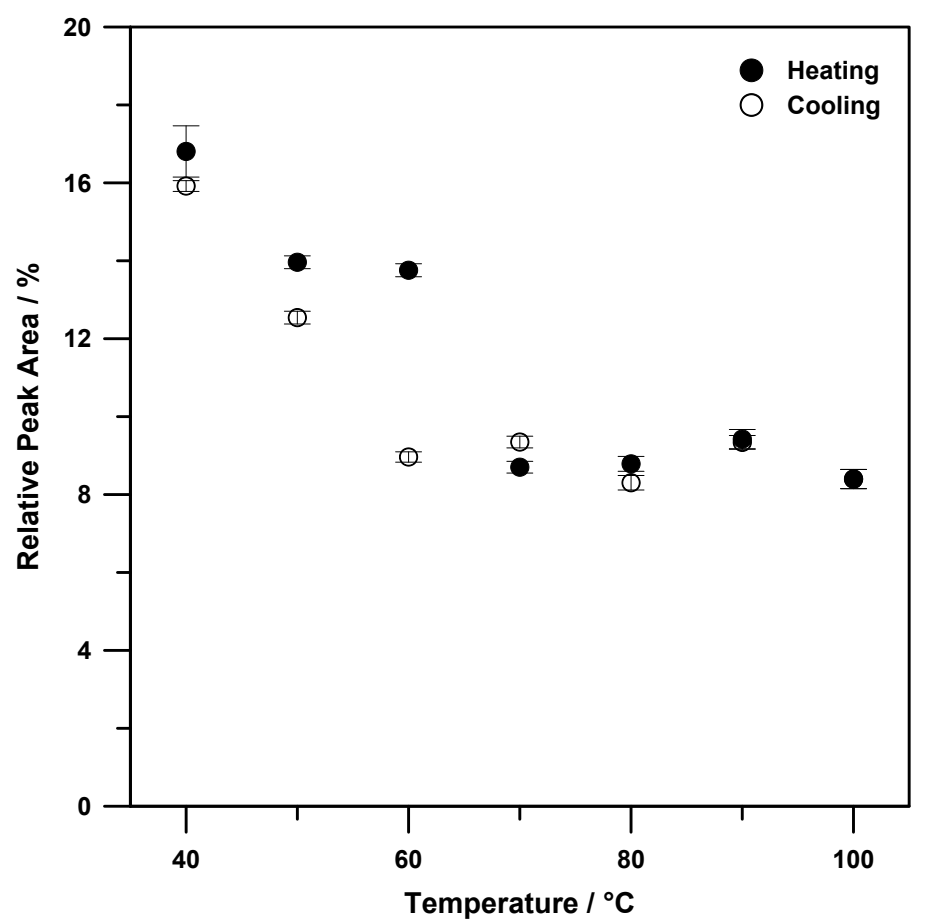

Figure S9. Relative area of the fatty acid cluster peak with respect to the area of peak attributed to the bulk liquid as a function of temperature on heating and on cooling for decanoic acid ( $T_{\text {fus }}=$ $32{ }^{\circ} \mathrm{C}$ ). The relative peak area does not reach 0 in this case because the threshold temperature for supercooling has not been passed.

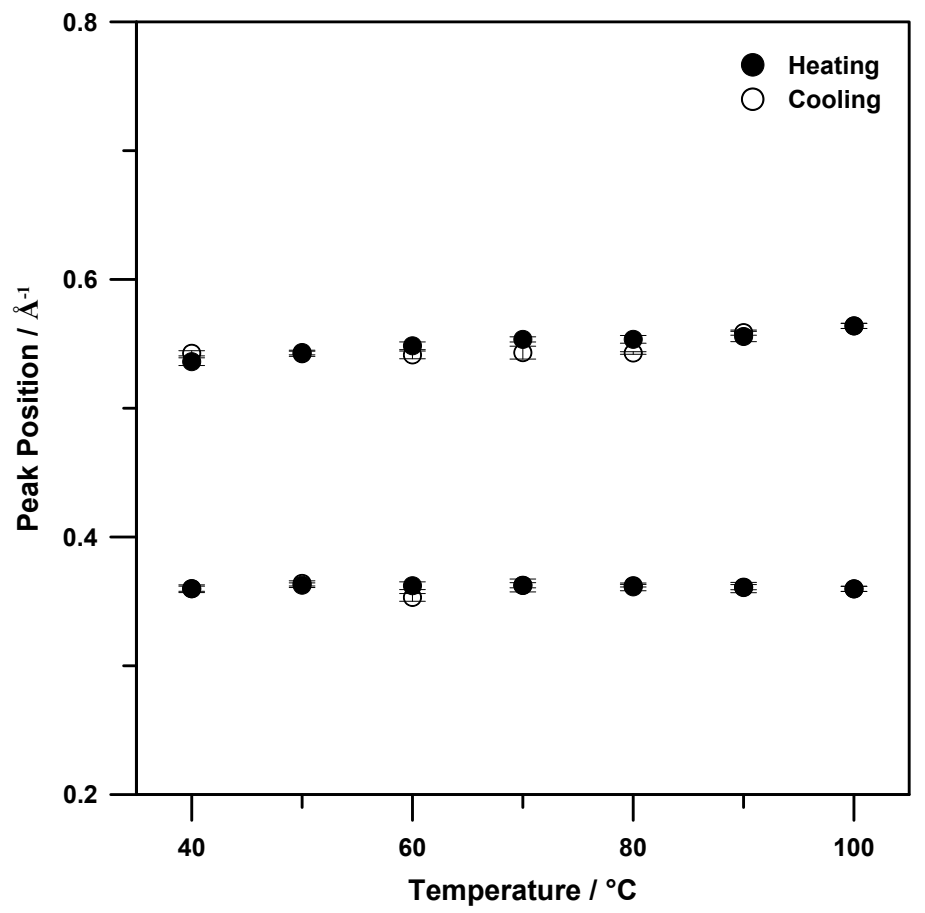

Figure S10. Peak positions of the SAXS diffraction peaks attributed to the bulk liquid and to fatty acid clusters for decanoic acid $\left(T_{\text {fus }}=32^{\circ} \mathrm{C}\right)$. 


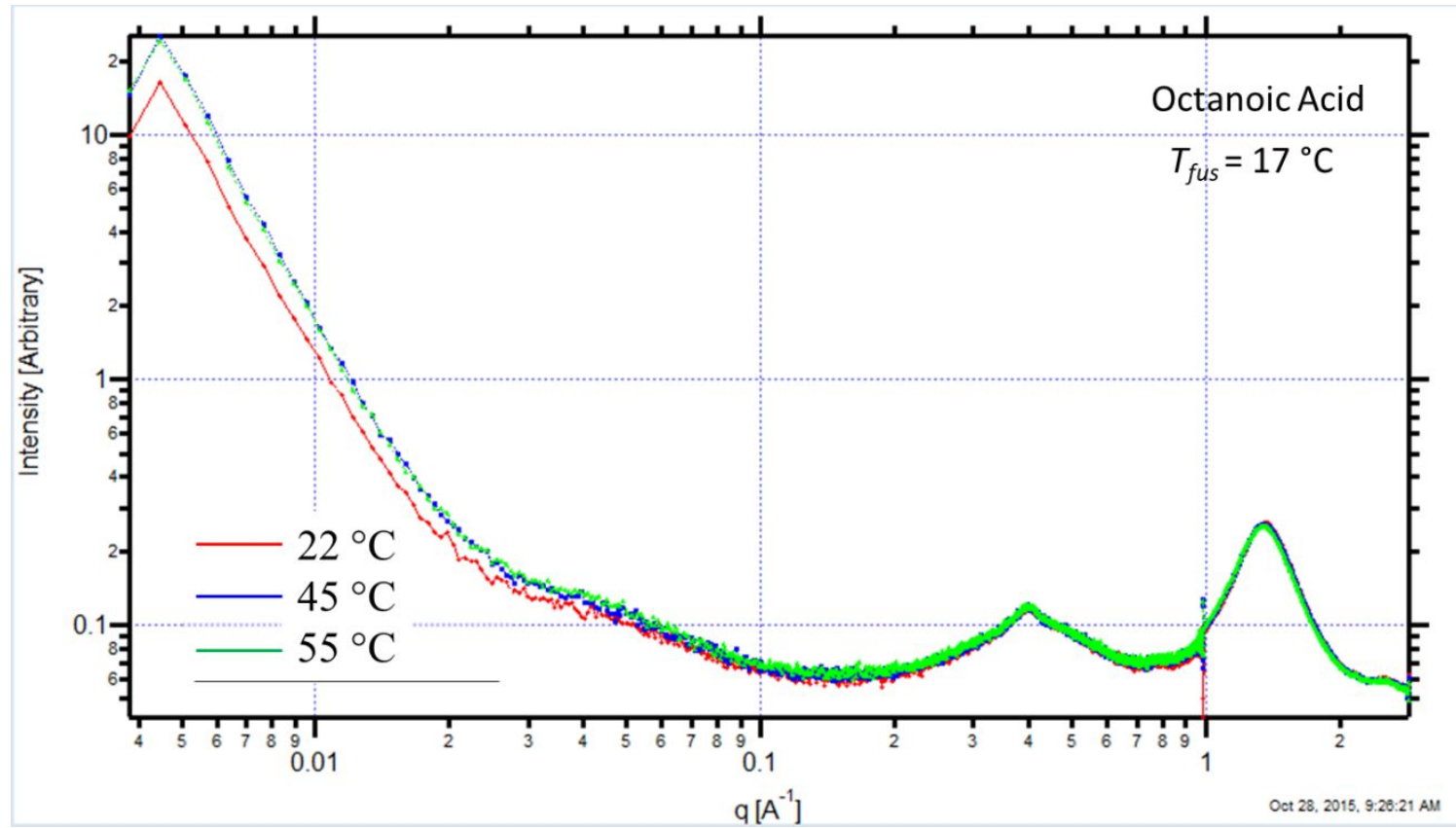

Figure S11. SAXS patterns of octanoic acid at three temperatures, acquired using synchrotron radiation. These patterns were used for Guinier analysis. 


\section{S.3. Crystallographic Coordinates and Cell Parameters for Structures Computed with Quantum ESPRESSO v. 5.1; B86bPBE-XDM}

S.3.1. Octanoic Acid Crystal - Herringbone

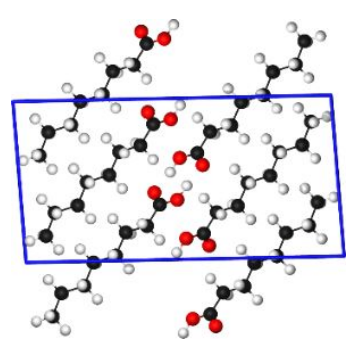

Figure S12. Octanoic Acid crystal in herringbone structure.

$\begin{array}{lrll}\text { ATOMIC POSITIONS (Crystal) } & \\ \mathrm{O} & 0.475499655 & 1.717121507 & 0.196858602 \\ \mathrm{H} & 0.452004884 & 1.788729977 & 0.091419298 \\ \mathrm{O} & 0.411049624 & 1.885911363 & 0.934026166 \\ \mathrm{C} & 0.552753855 & 1.890827113 & 0.185273212 \\ \mathrm{C} & 0.592870420 & 1.790977228 & 0.329819481 \\ \mathrm{H} & 0.605676060 & 1.571454505 & 0.278878363 \\ \mathrm{H} & 0.539991447 & 1.752660089 & 0.488725485 \\ \mathrm{C} & 0.677875229 & 1.996356812 & 0.334591610 \\ \mathrm{H} & 0.731914242 & 2.009940554 & 0.177739400 \\ \mathrm{H} & 0.334681641 & 1.780116916 & 0.633989442 \\ \mathrm{C} & 0.708967723 & 1.897664737 & 0.501732587 \\ \mathrm{H} & 0.716774842 & 1.667904186 & 0.477497417 \\ \mathrm{H} & 0.656150073 & 1.897546010 & 0.659446645 \\ \mathrm{C} & 0.202531304 & 1.908734012 & 0.501783367 \\ \mathrm{H} & 0.208151641 & 1.676190665 & 0.495415060 \\ \mathrm{H} & 0.148357561 & 1.929338039 & 0.654062807 \\ \mathrm{C} & 0.824939890 & 2.019719229 & 0.678841854 \\ \mathrm{H} & 0.770843560 & 2.042155170 & 0.830698712 \\ \mathrm{H} & 0.830843059 & 1.787597504 & 0.673953429 \\ \mathrm{C} & 0.086194566 & 1.783499257 & 0.329745075 \\ \mathrm{H} & 0.033721564 & 1.800028366 & 0.485117603 \\ \mathrm{H} & 0.093251363 & 1.552721872 & 0.321263158 \\ \mathrm{C} & 0.944919178 & 2.145630054 & 0.844563798 \\ \mathrm{H} & -0.010426728 & 1.715217027 & 0.171773496 \\ \mathrm{H} & 0.952070464 & 1.915697349 & 0.841867865 \\ \mathrm{H} & 0.103446277 & 1.820636641 & -0.001728963 \\ \mathrm{O} & 0.524500345 & 2.282878463 & 0.803141398 \\ \mathrm{H} & 0.547995116 & 2.211269993 & 0.908580702 \\ \mathrm{O} & 0.588950376 & 2.114088607 & 0.065973834 \\ \mathrm{C} & 0.447246145 & 2.109172857 & 0.814726788 \\ \mathrm{C} & 0.407129580 & 2.209022742 & 0.670180519 \\ \mathrm{H} & 0.394323940 & 2.428545465 & 0.721121637 \\ \mathrm{H} & 0.460008553 & 2.247339881 & 0.511274515 \\ \mathrm{C} & 0.322124771 & 2.003643158 & 0.665408390 \\ \mathrm{H} & 0.268085748 & 1.990059416 & 0.822260600 \\ \mathrm{H} & 0.665318359 & 2.219883054 & 0.366010558 \\ \mathrm{C} & 0.291032277 & 2.102335233 & 0.498267413 \\ \mathrm{H} & 0.283225158 & 2.332095784 & 0.522502583 \\ \mathrm{H} & 0.343849927 & 2.102453960 & 0.340553355 \\ \mathrm{C} & 0.797468696 & 2.091265958 & 0.498216633 \\ \mathrm{H} & 0.791848359 & 2.323809305 & 0.504584940 \\ \mathrm{H} & 0.851642439 & 2.070661931 & 0.345937193 \\ \mathrm{C} & 0.175060110 & 1.980280741 & 0.321158146\end{array}$

$\begin{array}{llll}\mathrm{H} & 0.229156440 & 1.957844800 & 0.169301288 \\ \mathrm{H} & 0.169156941 & 2.212402466 & 0.326046571 \\ \mathrm{C} & 0.913805434 & 2.216500713 & 0.670254925 \\ \mathrm{H} & 0.966278436 & 2.199971604 & 0.514882397 \\ \mathrm{H} & 0.906748637 & 2.447278098 & 0.678736852 \\ \mathrm{C} & 0.055080822 & 1.854369916 & 0.155436202 \\ \mathrm{H} & 1.010426728 & 2.284782943 & 0.828226504 \\ \mathrm{H} & 0.047929536 & 2.084302621 & 0.158132135 \\ \mathrm{H} & 0.896553723 & 2.179363319 & 1.001728963\end{array}$

Crystal axes: (Cart. Coord. in units of alat, bohr) $\mathrm{a}(1)=\left(\begin{array}{lll}30.018672 & -3.022235 & 6.484961\end{array}\right)$ $\mathrm{a}(2)=\left(\begin{array}{lll}-0.996250 & 8.736218 & -0.021472\end{array}\right)$ $\mathrm{a}(3)=\left(\begin{array}{lll}2.149818 & -0.264341 & 12.701967\end{array}\right)$

! total energy $=-1003.69119575 \mathrm{Ry}$

Harris-Foulkes estimate $=-1003.69119576 \mathrm{Ry}$ estimated scf accuracy $<\quad 1.7 \mathrm{E}-09$ Ry

total all-electron energy $=\quad-3722.817115 \mathrm{Ry}$

The total energy is the sum of the following terms:

One-electron Contribution $=-810.81634544 \mathrm{Ry}$ Hartree Contribution $=491.74418638 \mathrm{Ry}$ $\mathrm{xC}$ Contribution $\quad=-255.73803371 \mathrm{Ry}$ ewald Contribution $\quad=-151.88901602$ Ry Dispersion XDM Correction $=\quad-0.51897190 \mathrm{Ry}$ One-Center paw Contrib. $=-276.47301506 \mathrm{Ry}$ 
S.3.2. Octanoic Acid Crystal - Stacked

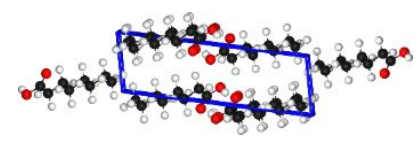

Figure S13. Octanoic acid crystal in stacked conformation.

\begin{tabular}{|c|c|c|c|}
\hline \multicolumn{4}{|c|}{ ATOMIC_POSITIONS (Crystal) } \\
\hline $\mathrm{O}$ & $0.4 \overline{9} 1065035$ & 0.248285685 & 0.370388914 \\
\hline & 0.508934965 & 0.748285685 & 0.129611086 \\
\hline & 0.508934965 & 0.751714315 & 0.629611086 \\
\hline & 0.491065035 & 0.251714315 & 0.870388914 \\
\hline $\mathrm{H}$ & 0.524329114 & 0.312571536 & 0.457322799 \\
\hline 1 & 0.475670886 & 0.812571536 & 0.042677201 \\
\hline & 0.475670886 & 0.687428464 & 0.542677201 \\
\hline $\mathrm{H}$ & 0.524329114 & 0.187428464 & 0.957322799 \\
\hline & 0.420626695 & 0.613845310 & 0.417795638 \\
\hline ) & 0.579373305 & 0.113845310 & 0.082204362 \\
\hline $\mathrm{O}$ & 0.579373305 & 0.386154690 & 0.582204362 \\
\hline 0 & 0.420626695 & 0.886154690 & 0.917795638 \\
\hline $\mathrm{C}$ & 0.434500768 & 0.411776741 & 0.342638139 \\
\hline $\mathrm{C}$ & 0.565499232 & 0.911776741 & 0.157361861 \\
\hline $\mathrm{C}$ & 0.565499232 & 0.588223259 & 0.657361861 \\
\hline $\mathrm{C}$ & 0.434500768 & 0.088223259 & 0.842638139 \\
\hline $\mathrm{C}$ & 0.388636059 & 0.326427960 & 0.211766902 \\
\hline $\mathrm{C}$ & 0.611363941 & 0.826427960 & 0.288233098 \\
\hline & 0.611363941 & 0.673572040 & 0.788233098 \\
\hline $\mathrm{C}$ & 0.388636059 & 0.173572040 & 0.711766902 \\
\hline $\mathrm{H}$ & 0.374667045 & 0.109018695 & 0.226166247 \\
\hline $\mathrm{H}$ & 0.625332955 & 0.609018695 & 0.273833753 \\
\hline H & 0.625332955 & 0.890981305 & 0.773833753 \\
\hline $\mathrm{H}$ & 0.374667045 & 0.390981305 & 0.726166247 \\
\hline $\mathrm{H}$ & 0.425161298 & 0.328758572 & 0.122954060 \\
\hline $\mathrm{H}$ & 0.574838702 & 0.828758572 & 0.377045940 \\
\hline $\mathrm{H}$ & 0.574838702 & 0.671241428 & 0.877045940 \\
\hline $\mathrm{H}$ & 0.425161298 & 0.171241428 & 0.622954060 \\
\hline $\mathrm{C}$ & 0.321007898 & 0.498799044 & 0.174539988 \\
\hline C & 0.678992102 & 0.998799044 & 0.325460012 \\
\hline C & 0.678992102 & 0.501200956 & 0.825460012 \\
\hline $\mathrm{C}$ & 0.321007898 & 0.001200956 & 0.674539988 \\
\hline $\mathrm{H}$ & 0.283313584 & 0.485453211 & 0.261241535 \\
\hline $\mathrm{H}$ & 0.716686416 & 0.985453211 & 0.238758465 \\
\hline $\mathrm{H}$ & 0.716686416 & 0.514546789 & 0.738758465 \\
\hline $\mathrm{H}$ & 0.283313584 & 0.014546789 & 0.761241535 \\
\hline & 0.336475482 & 0.715795819 & 0.166599892 \\
\hline $\mathrm{H}$ & 0.663524518 & 0.215795819 & 0.333400108 \\
\hline $\mathrm{H}$ & 0.663524518 & 0.284204181 & 0.833400108 \\
\hline & 0.336475482 & 0.784204181 & 0.666599892 \\
\hline $\mathrm{C}$ & 0.283491791 & 0.404943511 & 0.032224858 \\
\hline C & 0.716508209 & 0.904943511 & 0.467775142 \\
\hline & 0.716508209 & 0.595056489 & 0.967775142 \\
\hline $\mathrm{C}$ & 0.283491791 & 0.095056489 & 0.532224858 \\
\hline $\mathrm{H}$ & 0.274033842 & 0.181679883 & 0.036102186 \\
\hline $\mathrm{H}$ & 0.725966158 & 0.681679883 & 0.463897814 \\
\hline & 0.725966158 & 0.818320117 & 0.963897814 \\
\hline $\mathrm{H}$ & 0.274033842 & 0.318320117 & 0.536102186 \\
\hline $\mathrm{H}$ & 0.321070205 & 0.438922927 & 0.946621776 \\
\hline $\mathrm{H}$ & 0.678929795 & 0.938922927 & 0.553378224 \\
\hline $\mathrm{H}$ & 0.678929795 & 0.561077073 & 0.053378224 \\
\hline & 0.321070205 & 0.061077073 & 0.446621776 \\
\hline & 0.211394853 & 0.548911808 & 0.991740868 \\
\hline & 0.788605147 & 0.048911808 & 0.508259132 \\
\hline & 0.788605147 & 0.451088192 & 0.008259132 \\
\hline C & 0.211394853 & 0.951088192 & 0.491740868 \\
\hline $\mathrm{H}$ & 0.220454961 & 0.772252151 & 0.986790170 \\
\hline & 0.779545039 & 0.272252151 & 0.513209830 \\
\hline & 0.779545039 & 0.227747849 & 0.013209830 \\
\hline & 0.220454961 & 0.727747849 & 0.486790170 \\
\hline & 0.173298295 & 0.515939291 & 0.076719530 \\
\hline & 0.826701705 & 0.015939291 & 0.423280470 \\
\hline & & & \\
\hline
\end{tabular}

$\begin{array}{llll}\mathrm{H} & 0.173298295 & 0.984060709 & 0.576719530\end{array}$ $\begin{array}{lllll}\text { C } & 0.176576361 & 0.449083823 & 0.848089604\end{array}$ $\begin{array}{llll}\text { C } & 0.823423639 & 0.949083823 & 0.651910396\end{array}$ $\begin{array}{llll}\text { C } & 0.823423639 & 0.550916177 & 0.151910396\end{array}$ $\begin{array}{llll}\text { C } & 0.176576361 & 0.050916177 & 0.348089604\end{array}$ $\begin{array}{lllll}\mathrm{H} & 0.214325587 & 0.487238328 & 0.763000847\end{array}$ $\begin{array}{lllll}\mathrm{H} & 0.785674413 & 0.987238328 & 0.736999153\end{array}$ $\begin{array}{lllll}\mathrm{H} & 0.785674413 & 0.512761672 & 0.236999153\end{array}$ $\begin{array}{lllll}\mathrm{H} & 0.214325587 & 0.012761672 & 0.263000847\end{array}$ $\begin{array}{llll}\mathrm{H} & 0.169828927 & 0.224344188 & 0.852391212\end{array}$ $\begin{array}{lllll}\mathrm{H} & 0.830171073 & 0.724344188 & 0.647608788\end{array}$ $\begin{array}{lllll}\mathrm{H} & 0.830171073 & 0.775655812 & 0.147608788\end{array}$ $\begin{array}{llll}\mathrm{H} & 0.169828927 & 0.275655812 & 0.352391212\end{array}$ $\begin{array}{lllll}\text { C } & 0.102893378 & 0.579902680 & 0.805363374\end{array}$ $\begin{array}{llll}\text { C } & 0.897106622 & 0.079902680 & 0.694636626\end{array}$ $\begin{array}{llll}\text { C } & 0.897106622 & 0.420097320 & 0.194636626\end{array}$ $\begin{array}{lllll}\text { C } & 0.102893378 & 0.920097320 & 0.305363374\end{array}$ $\begin{array}{lllll}\mathrm{H} & 0.065178757 & 0.542717065 & 0.890519532\end{array}$ $\begin{array}{lllll}\mathrm{H} & 0.934821243 & 0.042717065 & 0.609480468\end{array}$ $\begin{array}{lllll}\mathrm{H} & 0.934821243 & 0.457282935 & 0.109480468\end{array}$ $\begin{array}{llll}\mathrm{H} & 0.065178757 & 0.957282935 & 0.390519532\end{array}$ $\begin{array}{lllll}\mathrm{H} & 0.109242106 & 0.804436909 & 0.799811654\end{array}$ $\begin{array}{lllll}\mathrm{H} & 0.890757894 & 0.304436909 & 0.700188346\end{array}$ $\begin{array}{lllll}\mathrm{H} & 0.890757894 & 0.195563091 & 0.200188346\end{array}$ $\begin{array}{lllll}\mathrm{H} & 0.109242106 & 0.695563091 & 0.299811654\end{array}$ $\begin{array}{llll}\text { C } & 0.069500540 & 0.473270223 & 0.662086039\end{array}$ $\begin{array}{lllll}\text { C } & 0.930499460 & 0.973270223 & 0.837913961\end{array}$ $\begin{array}{lllll}\text { C } & 0.930499460 & 0.526729777 & 0.337913961\end{array}$ $\begin{array}{lllll}\text { C } & 0.069500540 & 0.026729777 & 0.162086039\end{array}$ $\begin{array}{lllll}\mathrm{H} & 0.017016340 & 0.571416380 & 0.632609065\end{array}$ $\mathrm{H} \quad \begin{array}{llll}0.982983660 & 0.071416380 & 0.867390935\end{array}$ $\begin{array}{lllll}\mathrm{H} & 0.982983660 & 0.428583620 & 0.367390935\end{array}$ $\begin{array}{lllll}\mathrm{H} & 0.017016340 & 0.928583620 & 0.132609065\end{array}$ $\begin{array}{llll}\mathrm{H} & 0.060052453 & 0.250833824 & 0.666251053\end{array}$ $\begin{array}{lllll}\mathrm{H} & 0.939947547 & 0.750833824 & 0.833748947\end{array}$ $\begin{array}{lllll}\mathrm{H} & 0.939947547 & 0.749166176 & 0.333748947\end{array}$ $\begin{array}{llll}\mathrm{H} & 0.060052453 & 0.249166176 & 0.166251053\end{array}$ $\begin{array}{lllll}\mathrm{H} & 0.105702575 & 0.511846385 & 0.575001265\end{array}$ $\begin{array}{llll}\mathrm{H} & 0.894297425 & 0.011846385 & 0.924998735\end{array}$ $\begin{array}{lllll}\mathrm{H} & 0.894297425 & 0.488153615 & 0.424998735\end{array}$ $\begin{array}{lllll}\mathrm{H} & 0.105702575 & 0.988153615 & 0.075001265\end{array}$

Crystal axes: (Cart. Coord. in units of alat, bohr) $\mathrm{a}(1)=\left(\begin{array}{lll}34.739141 & 0.000000 & 0.703294\end{array}\right)$ $\mathrm{a}(2)=\left(\begin{array}{lll}0.000000 & 9.207619 & 0.000000\end{array}\right)$ $\mathrm{a}(3)=\left(\begin{array}{llll}-1.434602 & 0.000000 & 17.575586\end{array}\right)$

$!$ total energy $=-501.82362493 \mathrm{Ry}$ Harris-Foulkes estimate $=-501.82362493 \mathrm{Ry}$ estimated scf accuracy $<\quad 3.8 \mathrm{E}-09 \mathrm{Ry}$

total all-electron energy $=-1861.386585 \mathrm{Ry}$

The total energy is the sum of the following terms:

One-electron Contribution $=-383.61144111 \mathrm{Ry}$ Hartree Contribution $=231.91037953 \mathrm{Ry}$ $\mathrm{xC}$ Contribution $\quad=-127.83527274 \mathrm{Ry}$ ewald Contribution $=-83.82407560$ Ry Dispersion XDM Correction $=\quad-0.22695739$ Ry One-Center paw Contrib. $=-138.23625761 \mathrm{Ry}$ 


\title{
S.4. Cartesian Coordinates and Energies for Structures Computed with Gaussian 09 v. B01; B3LYP/6-31+G(d)-XDM
}

\author{
S.4.1. Octanoic Acid Monomer
}

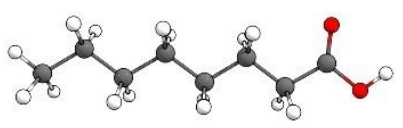

Figure S14. Octanoic acid monomer.

H 18.8865010002 .6792670005 .924388000 C 19.0684720002 .1316430003 .840539000 H 18.7326010001 .0924010003 .977550000 C 17.8950880002 .9778220003 .332246000 H 17.1117570003 .0096950004 .103384000 H 18.2309040004 .0159280003 .194978000 C 17.3002720002 .4550660002 .020386000 H 16.4644960003 .0789090001 .681741000 H 16.9257490001 .4298980002 .136803000 H 18.0531250002 .4435280001 .221719000 O 24.2490390001 .1628450009 .150139000 C 23.2156380001 .9545040008 .753546000 C 22.6064180001 .4648420007 .460655000 H 22.296655000 0.4219470007 .614776000 H 23.409256000 1.4223050006 .711666000 C 21.4386320002 .3219320006 .971341000 H 20.6631430002 .3541360007 .747003000 H 21.7786670003 .3571120006 .841545000 C 20.8429730001 .7999040005 .659244000 H 20.5069600000 .7607620005 .796212000 H 21.6268280001 .7676490004 .887225000 C 19.6701230002 .6470570005 .152690000 H 20.0060030003 .6858230005 .015695000 H 19.8524530002 .0992690003 .068574000
H 24.5904990001 .5364200009 .984508000 O 22.8646640002 .9230890009 .393677000

Temperature 298.150 Kelvin. Pressure 1.00000 Atm. Zero-point Correction $=0.233319$ (Hartree/Particle) Thermal Correction to Energy $=0.245767$ Thermal Correction to Enthalpy $=0.246711$ Thermal Correction to Gibbs Free Energy $=0.192600$ Sum of electronic and zero-point Energies $=-464.778515$ Sum Of electronic and Thermal Energies $=-464.766066$ Sum Of electronic and Thermal Enthalpies $=-464.765122$ Sum Of electronic and Thermal Free Energies $=-464.819234$ Sum Of electronic and XDM Energies $=-465.011833$ (Hartree/Particle)

\section{S.4.2. Octanoic Acid Dimer}

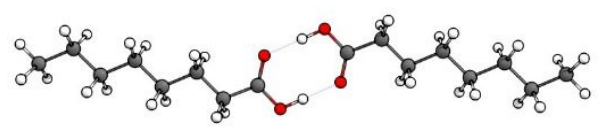

Figure S15. Octanoic acid dimer.

H $-6.712447000-0.990016000-0.875357000$ C -8.2500200000 .2517240000 .001732000$ H $-8.3739430000 .899724000-0.879353000$ C $-9.355106000-0.8112880000 .005237000$ $\mathrm{H}-9.232587000-1.462826000-0.872141000$ H $-9.231358000-1.4583050000 .885784000$ C $-10.765675000-0.2126610000 .004683000$ $\mathrm{H}-11.533954000-0.9950490000 .007219000$ $\mathrm{H}-10.9293750000 .412967000-0.882207000$ $\mathrm{H}-10.9281470000 .4175040000 .888582000$ O $-0.9034300001 .619069000-0.003203000$ C $-1.8250690000 .664690000-0.003343000$ C $-3.2350460001 .205279000-0.003764000$ H $-3.3307980001 .863239000-0.878583000$ H -3.3298150001 .8679100000 .867583000$ C $-4.3203550000 .128688000-0.000441000$ H $-4.190196000-0.521155000-0.875004000$ $\mathrm{H}-4.189278000-0.5166500000 .877334000$ C $-5.7317730000 .726228000-0.001193000$
H -5.855744000 $1.374080000-0.882299000$ H -5.8547070001 .3787550000 .876603000$ C $-6.835170000-0.3378320000 .002300000$ $\mathrm{H}-6.711265000-0.9854540000 .883163000$ H -8.3727420000 .9042740000 .879620000$ O $0.903330000-1.618727000-0.003193000$ H $0.0163310001 .217466000-0.003210000$ H $-0.016428000-1.217064000-0.003283000$ O $-1.558063000-0.537839000-0.003323000$ O $1.5581260000 .538138000-0.003060000$ C $1.825033000-0.664414000-0.003164000$ C $3.234973000-1.205117000-0.003477000$ H $3.329636000-1.8676680000 .867941000$ H $3.330735000-1.863175000-0.878224000$ C $4.320366000-0.128610000-0.000176000$ H 4.1894530000 .5166400000 .877692000 H $4.1901450000 .521344000-0.874644000$ C $5.731738000-0.726256000-0.001168000$ H $5.854672000-1.3790520000 .876427000$ 
H $5.855608000-1.373857000-0.882475000$ C 6.8352170000 .3377180000 .002587000 H $6.7123750000 .990315000-0.874745000$ H 6.7115290000 .9849440000 .883771000 C $8.250023000-0.2519450000 .001473000$ H $8.373777000-0.899419000-0.880022000$ H $8.372816000-0.9050370000 .878950000$ C 9.3551890000 .8109810000 .005477000 H 9.2316470001 .4574260000 .886471000 H 9.232561000 $1.463108000-0.871448000$ C 10.7657140000 .2122530000 .004274000 H 11.5340500000 .9945840000 .007210000 H $10.928293000-0.4185230000 .887718000$ H $10.929216000-0.412784000-0.883070000$

Temperature 298.150 Kelvin. Pressure 1.00000 Atm. Zero-point Correction $=0.468801$ (Hartree/Particle) Thermal Correction to Energy $=0.494957$

Thermal Correction to Enthalpy $=0.495901$ Thermal Correction to Gibbs Free Energy= 0.404853

Sum Of electronic and zero-point Energies $=-929.584194$

Sum Of electronic and Thermal Energies $=-929.558039$

Sum Of electronic and Thermal Enthalpies $=-929.557095$

Sum Of electronic and Thermal Free Energies $=$-929.648143

Sum Of electronic and XDM Energies $=-930.052530$ (Hartree/Particle)

\section{S.4.3. Octanoic Acid Catamer}

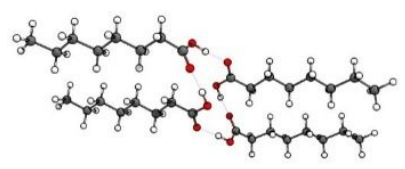

Figure S16. Octanoic acid catamer.

H $8.057126000-4.2393040000 .572952000$ C $9.550702000-3.208584000-0.606378000$ H $10.194029000-4.087490000-0.732628000$ H $9.959657000-2.6096860000 .217227000$ H 2.9447210000 .0541270001 .496061000 H $2.490741000-0.073086000-0.190120000$ C $3.344418000-1.8961160000 .618830000$ H $2.920911000-2.531874000-0.167775000$ C $4.805488000-1.5570930000 .301615000$ H $5.210475000-0.9188460001 .100847000$ H $4.848798000-0.952943000-0.616671000$ C $5.710506000-2.7821180000 .137353000$ H $5.328385000-3.412650000-0.679089000$ H 5.662446000 -3.397433000 1.048179000 C $7.169416000-2.405224000-0.146537000$ H $7.212538000-1.776821000-1.048913000$ H $7.548036000-1.7784400000 .674436000$ C $8.098125000-3.610747000-0.328449000$ H $7.725171000-4.234813000-1.153427000$ H $9.625695000-2.605474000-1.520303000$ O $1.9480480002 .840814000-1.070971000$ H $1.0258610002 .437968000-1.102148000$ C 2.4343710002 .8822460000 .158805000 C 3.8256530003 .4629810000 .231842000 H $3.9700960004 .174997000-0.586586000$ H 3.9338980003 .9865340001 .186892000 C 6.3132970002 .8095460000 .133727000 H $6.4735200003 .487972000-0.716923000$ H 6.5053430003 .3997080001 .041949000 C 8.7767810002 .0777320000 .030007000
H 8.9991120002 .6766300000 .926189000 H $8.9463990002 .741606000-0.831182000$ C $11.2203070001 .321583000-0.074467000$ H $11.4291510001 .957206000-0.944550000$ O 0.3274800000 .2066420001 .453440000 O $-1.947586000-2.8413830001 .073292000$ H 0.8349670001 .0574800001 .342048000 H - $1.025392000-2.4385730001 .104206000$ O $0.552772000-1.9781090001 .157949000$ O $-1.853595000-2.428375000-1.144779000$ C $1.059105000-0.8611120001 .140073000$ C - $2.434390000-2.882622000-0.156263000$ C $2.503164000-0.6268390000 .758858000$ C $-3.825724000-3.463298000-0.228881000$ H $-3.933953000-3.987698000-1.183471000$ H $-3.970348000-4.1745570000 .590167000$ C $-4.861100000-2.322869000-0.132352000$ H $3.288136000-2.4782290001 .547391000$ H $-4.709375000-1.636182000-0.973864000$ H $-4.673909000-1.7459580000 .782912000$ C $-6.313292000-2.809466000-0.131607000$ H $-6.505349000-3.400345000-1.039360000$ $\mathrm{H}-6.473632000-3.4871910000 .719578000$ C $-7.307510000-1.644197000-0.056222000$ H -7.091306000-1.043095000 0.839060000 H $-7.144149000-0.975365000-0.913743000$ C $-8.776706000-2.077356000-0.028505000$ H $-8.999094000-2.676972000-0.924193000$ H $-9.577800000-0.232355000-0.817513000$ O - $0.5521760001 .977140000-1.156708000$ 
H $-3.2874340002 .477581000-1.548666000$ C $-5.7106200002 .782388000-0.140381000$ H -5.661921000 $3.397187000-1.051522000$ C -8.0984260003 .6115900000 .323382000$ $\mathrm{H}-7.7259130004 .2361310001 .148200000$ H -8.056775000 $4.239570000-0.578392000$ H -10.1945220004 .0888660000 .725921000$ H -9.959742000 2.610419000 -0.222833000 O 1.8531740002 .4280280001 .147075000 C 4.8611600002 .3227820000 .134159000 H $4.6739400001 .746662000-0.781591000$ H 4.7096360001 .6353170000 .975074000 C 7.3076210001 .6444310000 .057365000 H 7.144321000 0.9748760000 .914332000 H $7.0914610001 .044048000-0.838410000$ C $9.7496130000 .894203000-0.043276000$ H $9.5223400000 .293856000-0.935406000$ H 9.5780010000 .2321380000 .817506000

H $11.8885000000 .453804000-0.127528000$ H 11.4857690001 .8931670000 .824164000 O $-0.327333000-0.207717000-1.452106000$ $\mathrm{H}-0.835404000-1.058383000-1.341659000$ C $-1.0588120000 .860269000-1.139189000$ C $-2.5031950000 .626405000-0.758898000$ $\mathrm{H}-2.4915250000 .0729880000 .190289000$ $\mathrm{H}-2.944382000-0.054762000-1.496131000$ C $-3.3443370001 .895864000-0.619891000$ $\mathrm{H}-2.9212060002 .5318940000 .166694000$ C $-4.8056420001 .557169000-0.303405000$ $\mathrm{H}-4.8495880000 .9535240000 .615183000$ $\mathrm{H}-5.2101970000 .918526000-1.102542000$ H -5.3289270003 .4133320000 .675943000$ C -7.1697520002 .4058380000 .142816000$ $\mathrm{H}-7.5479240001 .778587000-0.678007000$ $\mathrm{H}-7.2135230001 .7780050001 .045557000$ C -9.5512320003 .2097940000 .600646000$ $\mathrm{H}-9.6268860002 .6072850001 .514913000$ H $-8.946370000-2.7405120000 .833228000$ C $-9.749444000-0.8936920000 .043823000$ H $-9.522099000-0.2926250000 .935448000$ C $-11.220173000-1.3209190000 .075406000$ $\mathrm{H}-11.888286000-0.4530360000 .127767000$ H $-11.485710000-1.893224000-0.822744000$

H - $11.429050000-1.9558020000 .946021000$

Temperature 298.150 Kelvin. Pressure 1.00000 Atm. Zero-point Correction $=0.940568$ (Hartree/Particle) Thermal Correction to Energy $=0.994192$

Thermal Correction to Enthalpy $=0.995136$ Thermal Correction to Gibbs Free Energy $=0.838957$

Sum of electronic and zero-point Energies $=-1859.175761$ Sum of electronic and Thermal Energies $=-1859.122137$ Sum of electronic and Thermal Enthalpies $=-1859.121193$ Sum of electronic and Thermal Free Energies $=-1859.277372$

Sum Of electronic and XDM Energies $=-1860.116329$ (Hartree/Particle) 


\section{References Cited:}

${ }^{1}$ Noël, J. A.; Kreplak, L.; Getangama, N. N.; de Bruyn, J. R.; White, M. A. Supercooling and nucleation of fatty acids: Influence of thermal history on the behaviour of the liquid phase. $J$. Phys. Chem. B 2018, 122, 12386-12395.

${ }^{2}$ Abate, L.; Badea, E.; Blanco, I.; Della Gatta, G. Heat capacities and enthalpies of solid-solid transitions and fusion of a series of eleven primary alkylamides by differential scanning calorimetry. J. Chem. Eng. Data. 2008, 53, 959-956.

3 Paolantoni, M.; Sassi, P.; Morresi, A.; Cataliotti, R. S. Infrared study of 1-octanol liquid structure. Chem. Phys. 2005, 310, 169-178.

${ }^{4}$ Iwahashi, M.; Hayashi, Y.; Hachiya, N.; Matsuzawa, H.; Kobayashi, H. Self-association of octan-1-ol in the pure liquid state and in decane solutions as observed by viscosity, selfdiffusion, nuclear magnetic resonance and near-infrared spectroscopy measurements. $J$. Chem. Soc. Faraday Trans. 1993, 89, 707-712.

${ }^{5}$ Bellamy, L. J.; Pace, R. J. Hydrogen bonding by alcohols and phenols - I. The nature of the hydrogen bond in alcohol dimers and polymers. Spectrochim. Acta. 1966, 22, 525-533.

${ }^{6}$ Iwahashi, M.; Hachiya, N.; Hayashi, Y.; Matsuzawa, H.; Liu, Y.; Czarnecki, M. A.; Ozaki, Y.; Horiuchi, T.; Suzuki, M. Self-association of cis-9-octadecen-1-ol in the pure liquid state and in decane solutions as observed by viscosity, self-diffusion, nuclear magnetic resonance, electron spin resonance, and near-infrared spectroscopic measurements. J. Phys. Chem. 1995, 99, 41554161. 